\title{
C Research Square

\section{A numerical feasibility study on monitoring bone health using microwave tomography: towards a wearable design}

Mohanad Alkhodari ( $\sim$ mohanad.alkhodari@ku.ac.ae)

Khalifa University https://orcid.org/0000-0002-5248-6327

Amer Zakaria

American University of Sharjah

Nasser Qaddoumi

American University of Sharjah

\section{Research}

Keywords: Microwave tomography, numerical analysis, wearable system, two-dimensional imaging, finiteelement method, contrast-source inversion, k-means clustering, bone degradation, bone health monitoring

Posted Date: June 29th, 2021

DOI: https://doi.org/10.21203/rs.3.rs-637448/v1

License: (a) (1) This work is licensed under a Creative Commons Attribution 4.0 International License. Read Full License 


\section{Abstract}

Background: Osteoporosis is the major cause of bone weakness and fragility in more than 10 million people in the United States. This disease causes bone fractures in the hip or spine, which result in increasing the risk of disabilities or even death. The current gold standard in osteoporosis diagnostics, Xray, although reliable, it uses ionizing radiations that makes it unfeasible for early and continuous monitoring applications. Recently, microwave tomography (MWT) has been emerging as a biomedical imaging modality that utilizes non-ionizing electromagnetic signals to screen bones' electrical properties. These properties are highly correlated to bones' density, which makes MWT to be an effective and safe alternative for frequent testing in osteoporosis diagnostics.

Results: Both of the conventional and wearable simulated systems were successful in localizing the tibia and fibula bones in the enhanced MWT images. Furthermore, structure extraction of the leg's model from the blind MWT images had a minimal error compared to the original one (L2-norm: 15.60\%). Under five sequentially-incremental bone volume fraction (BVF) scenarios simulating bones' treatment procedure, bones were detected successfully and their density were found to be inversely proportional to the real-part of the relative permittivity values.

Conclusions: This study paves the way towards implementing a safe and user-friendly MWT system that can be wearable to monitor bone degradation or treatment for osteoporosis cases.

Methods: An anatomically-realistic finite-element (FE) model representing the human leg was initially generated and filled with corresponding tissues' (skin, fat, muscles, and bones) dielectric properties. Then, numerically, the forward and inverse MWT problems were solved within the framework of the finiteelement method contrast source inversion algorithm (FEM-CSI). Furthermore, image reconstruction enhancements were investigated by utilizing prior information about different tissues as an inhomogeneous background as well as by adjusting the imaging domain and antennas locations based on the prior structural information. In addition, the utilization of a medically-approved matching medium that can be used in wearable applications, namely an ultrasound gel, was suggested. Additionally, an approach based on k-means clustering was developed to extract the prior structural information from blind reconstructions. Lastly. the enhanced images were used to monitor variations in BVF.

\section{Full Text}

This preprint is available for download as a PDF. 